\title{
A Randomized Controlled Trial: Attachment-Based Family and Nondirective Supportive Treatments for Youth Who Are Suicidal
}

\author{
Guy S. Diamond, PhD, R. Roger Kobak, PhD, E. Stephanie Krauthamer Ewing, PhD, MPH, \\ Suzanne A. Levy, PhD, Joanna L. Herres, PhD, Jody M. Russon, PhD, Robert J. Gallop, PhD
}

Objective: To evaluate the efficacy of attachment-based family therapy (ABFT) compared with a family-enhanced nondirective supportive therapy (FE-NST) for decreasing adolescents' suicide ideation and depressive symptoms.

Method: A randomized controlled trial of 129 adolescents who are suicidal ages 12- to 18-years-old (49\% were African American) were randomized to ABFT $(n=66)$ or FE-NST $(n=63)$ for 16 weeks of treatment. Assessments occurred at baseline and 4, 8, 12, and 16 weeks. Trajectory of change and clinical recovery were calculated for suicidal ideation and depressive symptoms.

Results: There was no significant between-group difference in the rate of change in self-reported ideation (Suicidal Ideation Questionnaire-Jr; $F_{1,127}=$ $181, p=.18)$. Similar results were found for depressive symptoms. However, adolescents receiving ABFT showed a significant decrease in suicide ideation $\left(t_{127}=12.61, p<.0001\right.$; effect size, $\left.d=2.24\right)$. Adolescents receiving FE-NST showed a similar significant decrease $\left(t_{127}=10.88, p<.0001\right.$; effect size, $d=1.93$ ). Response rates (ie, $\geq 50 \%$ decrease in suicide ideation symptoms from baseline) at post-treatment were $69.1 \%$ for ABFT versus $62.3 \%$ for FE-NST.

Conclusion: Contrary to expectations, ABFT did not perform better than FE-NST. The 2 treatments produced substantial decreases in suicidal ideation and depressive symptoms that were comparable to or better than those reported in other more intensive, multicomponent treatments. The equivalent outcomes could be attributed to common treatment elements, different active mechanisms, or regression to the mean. Future studies will explore long-term follow up, secondary outcomes, and potential moderators and mediators.

Clinical trial registration information: Attachment-Based Family Therapy for Suicidal Adolescents; http://clinicaltrials.gov; NCT01537419.

Key words: suicide, depression, attachment, treatment

J Am Acad Child Adolesc Psychiatry 2019;58(7):721-731.

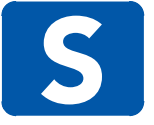

uicide is the third leading cause of death in American adolescents, accounting for 4,600 deaths each year. In addition, nearly 1 million adolescents attempt suicide each year, resulting in roughly 500,000 emergency room admissions. ${ }^{1}$ Adolescent suicide results in high emotional costs for families and financial costs for the health care system. Using broad selection criteria, a recent review found 18 randomized control trials (RCTs) that examined psychosocial (with or without medication) treatments for adolescents with suicide ideation or behaviors. ${ }^{2}$ Only 6 of these treatments met criteria for being probably or possibly efficacious. Furthermore, nearly all these treatments were supported by randomized trials that compared the treatment of interest with treatment as usual (TAU) in the community. These TAU comparison groups are highly variable in the number of sessions and nature of treatment received. As a result, interpretations of findings from studies using TAU as a comparison group are tenuous. ${ }^{3}$ Surprisingly, there have been few RCTs for adolescents who are suicidal that have compared 2 manualbased, supervised, monitored treatments provided within the same research center. The present study tested the hypothesis that attachment-based family therapy $(\mathrm{ABFT})^{4}$ would perform better than a manual-based commonly used nondirective individual supportive therapy ${ }^{5}$ enhanced with a parent education program.

ABFT has been designated as a probably efficacious treatment for suicidal thoughts and behaviors and for depression and depressive symptoms ${ }^{2}$ and is listed on the National Registry of Evidence-based Program and Practices for suicidal thoughts and behaviors and depression. ABFT provides a manual-based series of sequenced tasks, occurring 
Sophia is a 16-year-old white girl currently in the 10th grade. Sophia has never met her father. When Lisa, Sophia's mother, lost her job, they moved in with the maternal grandmother. Sophia was 10 years old. Lisa become depressed and through a new boyfriend became addicted to oxycodone. When Sophia was 13, Lisa moved in with her boyfriend and left Sophia with her grandmother. Lisa visited sporadically but eventually got arrested and went to a rehab program for 9 months. Six months after discharge, Lisa was stable and moved back into the home with the grandmother and Sophia. During Lisa's absence, Sophia began to act out, experimenting with drugs and risky sexual behavior. When a friend's mother discovered this, it led to a referral to a therapy program. At Sophia's intake, it became clear that her drug use and sexual behaviors were her best attempt at coping with her depression and suicidal impulses. Wanting to resume parenting of her daughter, Lisa brought Sophia to the first session, which infuriated Sophia. After some history gathering and assessment of the depression, the therapist focused on the mother-daughter relationship. "So Sophia, when you feel so bad, like you want to kill yourself, why don't you turn to your mother for help?" Sophia was reluctant to talk. Lisa resentfully said that Sophia thought she was all grown up and independent. Sophia said, "She does her thing and I do mine. I don't need her anymore." Because it was the first session, the therapist did not explore this in depth but empathically remarked that the distance between them was apparent and tragic. Commenting how disjointing this must feel, Lisa began to cry and Sophia looked on scornfully. The therapist said that maybe if they could work through some of these past hurts, they could be close again or at least not carry around such guilt (mother) and resentment (daughter). The family agreed to consider this attachment-focused treatment plan.

In a session alone with Sophia, the therapist explored her depression, suicidal feelings, and risky sexual behavior. The goal was not to change it (yet) but to help Sophia acknowledge for herself how miserable she felt and to make this her problem rather than everyone else's. In another session, the discussion focused on her mother. With a gentle directive approach, Sophia began to broaden her attachment narrative. Rather than just feel rage at her "selfish mother," Sophia allowed herself to feel the shame and disappointment of abandonment. She began to understand how feeling worthless and rejected by her mother allowed her to let boys to take advantage of her. When the timing was right in the third session alone, the therapist proposed that expressing her anger directly to her mother might help rid her of these ghosts of self-hatred. Sophia reluctantly agreed, and during the next session the therapist helped prepare Sophia for this conversation. Simultaneously, the therapist was having individual sessions with Lisa. Several sessions explored her current stressors related to getting her life back on track. One session focused on her relationship with her parents. The loss of her father when she was 11 devastated her, and her own mother's grief and withdrawal felt rejecting. Helping Lisa remember and feel her own experience of abandonment gave the therapist the opportunity to help her more empathically understand how Sophia might have felt when she left. Lisa, preoccupied with her own recovery, never really stopped to consider the impact her drug abuse had on her daughter. This moment of empathic sensitivity, of reflective functioning, motivated Lisa to let the therapist teach her some different, more emotion-focused, parenting skills.

At approximately week 8, the therapist brought the mother and daughter back together. Sophia expressed more directly, and in a more regulated manner, her hurt and feelings of abandonment. Lisa, rather than being defensive, empathically listened and encouraged Sophia to share these feelings and memories with her. When the timing was right, Lisa apologized for her actions. Sophia listened but did not feel moved to forgive her. Lisa had to accept that. However, when leaving the session, Sophia did allow her mother to put her arm around her shoulder. Two more sessions focused on helping the family discuss these past traumas and injustice. The grandmother participated in 1 of these sessions. As the tension at home dissipated, Sophia's depression lessened. Problems persisted with weekend drinking and Lisa had to increase her monitoring and setting of limits. Sophia resented this at first. The therapist helped frame this as her mother not wanting to abandon Sophia again by ignoring these problems. Sophia became more receptive when limits were viewed as protection rather than punishment. At the end of the short-term treatment (16 weeks), Sophia's depression and suicide ideation were out of clinical range. Then, the therapist referred Sophia to a dialectical behavior therapist to help her develop more effective emotion regulation sills. in individual and family sessions, that aim to identify and resolve attachment ruptures that have compromised adolescents' trust in their parents (eg, divorce, parental psychopathology, critical parenting). Working through these ruptures can increase the adolescent's confidence in a parents' availability $^{6}$ and increase the adolescent's capacity for problem solving and ability to use the parent as a resource for regulating affect and managing suicidal thoughts and feelings.
When adolescents view parents as sensitive, safe, and available, they are more likely to turn to parents for support that can buffer against common triggers for depressive feelings and suicide ideation (eg, grief and loss, bullying, school failure, romantic relationships, and family conflict). ${ }^{7}$ Studies have demonstrated that ABFT is more effective for this population than waitlist control or TAU. ${ }^{8}$ ABFT also has been successfully disseminated to community settings. ${ }^{9,10}$ 
Nondirective supportive therapy (NST) has been used as an active control condition in treatments of adolescent and adult depression. In NST, the central goal is to augment the adolescent's access to supportive adult relationships through the adolescent's relationship with the therapist. Therapists implement NST by focusing on reflective listening, empathizing with the adolescent's experiences of stress, and supporting the adolescent in articulating and exploring thoughts and feelings. In a study of adolescents with major depressive disorder, cognitivebehavioral therapy (CBT) proved more effective than systemic behavior family therapy or NST in decreasing depressive symptoms. ${ }^{5,11}$ However, Brent et al. ${ }^{5}$ reported equivalent decreases in suicidal symptoms and functional impairment in the NST, CBT, and systemic behavior family therapy conditions. In another study, NST produced equivalent decreases in suicide ideation and depressed mood compared with a directive skills-based protocol. ${ }^{11}$ The effectiveness of NST also extends to treatments of adult depression. In a meta-analysis of 31 studies on treatments for adult depression, NST was as effective as other active treatments, ${ }^{12}$ particularly when controlling for investigator allegiances. In this way, NST provides a rigorous test of the active ingredients of ABFT by controlling for a supportive therapeutic relationship and reflective listening as nonspecific factors of therapy.

To test the superiority of ABFT against NST, we randomized adolescents who are suicidal and their families to the 2 treatment conditions. Many characteristics of this RCT were designed to control for common treatment elements in ABFT and NST. First, a 5-session parent psychoeducation program was added to the NST condition to control for parent involvement in ABFT. Thus, we refer to the treatment used in this RCT as family-enhanced NST (FE-NST). Second, all patients received safety planning. Third, therapists implemented ABFT and FE-NST to control for therapist effects. Fourth, with the exception of 4 cases, treatment was implemented by trained community therapists. Fifth, treatment dosage was controlled by delivering the 2 treatments for 16 weeks with occasional 2 weekly sessions to incorporate parent sessions. Sixth, severe and persistent suicidal ideation and moderate depression were used as inclusion criteria. These criteria establish a common clinical presentation and increased risk for suicide attempts. ${ }^{13,14}$ Seventh, this study incorporated a diverse racial and ethnic sample, providing a test of the generalizability of treatment effects across race and social class. Suicide rates for minority youth have increased, ${ }^{15}$ yet minorities remain under-represented in clinical trials. ${ }^{16}$

\section{METHOD}

\section{Participants}

Participants were recruited from emergency departments (37.9\%), inpatient psychiatric hospitals (6.9\%), mental health agencies or primary care sites $(20.9 \%)$, schools $(10.8 \%)$, and community clinicians or self-referrals $(23.5 \%$; Figure 1). Eligibility for the study included at least clinically significant levels of suicidal ideation (Suicidal Ideation Questionnaire-Junior [SIQ-JR] score $\geq 31^{17}$ ) and moderate levels of depressive symptoms (Beck Depression Inventory II [BDI-II] score $>20^{17}$ ). Participants were screened using a multigate procedure over 2 pre-randomization time points. Participants were screened using the BDI-II and SIQ-JR, first over the phone and then at a face-to-face clinic visit, usually within 72 hours of the initial screening. To be eligible, participants were required to meet inclusion criteria at the 2 pretreatment screens. At least 1 primary caregiver was required to participate in assessments and treatments.

Exclusion criteria included imminent risk of harm to self or others that could not be safely treated on an outpatient basis; psychotic features; severe cognitive impairment based on educational records, parent report, and/or clinical impression; and non-English-speaking participating parent. Participants who began psychiatric medication within 3 weeks of the initial pretreatment screening also were ineligible to participate. The protocol was approved by the institutional review boards at the Children's Hospital of Philadelphia, Drexel University, and the City of Philadelphia. The study was monitored quarterly by a data safety and monitoring board. Participants were enrolled from May 1, 2012 through December 31, 2015 and provided written informed consent or assent $(<14$ years of age).

Participants included 129 adolescents (81.9\% girls) 12 to 18 years old (mean 14.87, SD 1.68). Sixty-four adolescents identified as African American (49.7\%), 37 as white (28.7\%), 3 as Asian (2.3\%), 2 as American Indian or Alaskan Native (1.6\%), and 1 as Native Hawaiian or Pacific Islander $(0.8 \%)$. Ten adolescents identified as biracial or multiracial (7.8\%) and 12 identified as "other" (9.3\%). Most of the sample identified as non-Hispanic/Latino $(84.5 \%)$ and $31 \%$ of adolescents identified as gay, lesbian, or bisexual. Parents reported that $69 \%$ were singleheaded households and $31 \%$ had incomes below the poverty line (Table 1). At baseline, the average score for suicidal ideation on the SIQ-JR was 49.89 (SD 15.2), and the average score on the BDI was 30.5 (SD 7.97; Table 2). Forty-two percent of participants had made at least 1 suicide attempt in their lifetime, $25 \%$ had a history of psychiatric hospitalization, and $57.5 \%$ reported a history of 


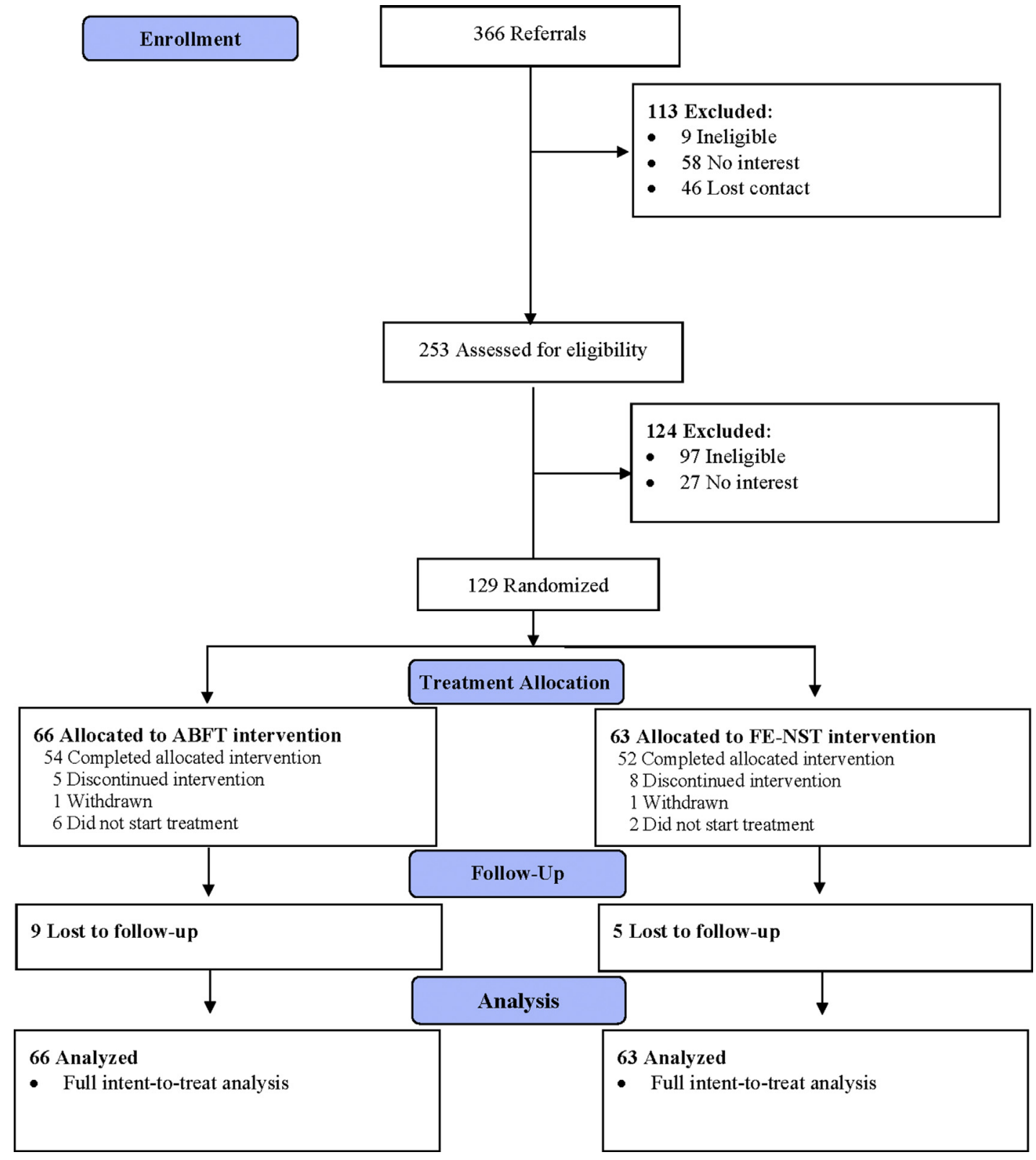

Note: ABFT = attachment-based family therapy; FE-NST = family-enhanced nondirective supportive therapy

nonsuicidal self-injury as indicated by the ColumbiaSuicide Severity Rating Scale (C-SSRS). ${ }^{18}$ At intake, $41.2 \%$ met criteria for major depressive disorder, $3.9 \%$ met criteria for dysthymia, and $46.93 \%$ met criteria for an anxiety disorder on the Diagnostic Interview Schedule for Children-IV (DISC-IV). ${ }^{18}$ At the time of intake, $27.1 \%$ of participants were currently taking medication for depression and $9.3 \%$ were taking medication for other behavioral or emotional problems.

\section{Procedures}

After the baseline assessment, urn randomization assigned participants to 16 weeks of ABFT or NST. Stratification variables included gender, history of attempt, and endorsement of high levels of family conflict (score $>13$ on the Conflict subscale of the Self-Report of Family Functioning $\left.[\mathrm{SRFF}]^{19}\right)$. Outcomes assessments were administered at weeks 0 (baseline), 4, 8, 12, and 16 (post-treatment assessment). Follow-up assessments were completed at 
TABLE 1 Frequencies for Sample Demographic Characteristics ( $N=129)$

\section{Variable}

Race

American Indian/Alaskan Native

Asian

White

African American

Native Hawaiian/Pacific Islander

Biracial/multiracial

Other

Hispanic

Relationship status

Single

In relationship

Physical abuse

Sexual abuse

Religion

Catholic

Other Christian

Jewish

Muslim

Buddhist

Hindu

Atheist

Other

Gender

Female

Male

Age

Age $\leq 15$ y

Sexual orientation

Heterosexual

Lesbian/gay

Bisexual

Questioning

Per capita ratio

Below poverty line

Prior suicide attempt (yes/no)

History of NSSI (yes/no) $(n=126)$

Any mood disorder $(n=113)$

Any anxiety disorder ( $\mathrm{n}=113$ )

Any substance disorder $(\mathrm{n}=112$ )

Oppositional defiant disorder

$(n=113)$
Total $(\mathbf{N}=129)$

Frequency $\%$

2

3

37

64

1

10

12

20

89

36

23

25

28

45

3

5

2

1

15

30

95

22

76

88

10

22

9

40

51

73

50

53

11

11

2.3

28.7

49.6

0.8

7.8

9.3

15.5

69.0

27.9

17.8

19.4

21.7

34.9

2.3

3.9

1.6

0.8

11.6

23.3

81.9

18.1

58.9

68.2

7.8

17.1

7.0

31.3

39.5

57.9

44.2

46.9

9.8

9.8
ABFT $(n=66)$

Frequency $\%$

$\begin{array}{rr}0 & 0.0 \\ 2 & 3.0 \\ 21 & 31.8 \\ 31 & 47.0 \\ 0 & 0.0 \\ 6 & 9.1 \\ 6 & 9.1 \\ 11 & 16.7\end{array}$

68.2

28.8

13.6

19.7

19.7

36.4

1.5

7.6

1.5

1.5

13.6

18.2

83.3

16.7

57.1

57.6

12.1

19.7

10.6

32.3

43.9

48.4

48.2

48.2

12.5

5.4
NST (n = 63)

Frequency $\%$

$x^{2}$

3.09

$\begin{array}{rr}2 & 3.2 \\ 1 & 1.6 \\ 16 & 25.4 \\ 33 & 52.4 \\ 1 & 1.6 \\ 4 & 6.3 \\ 6 & 9.5 \\ 9 & 14.3\end{array}$

0.14

0.53

69.8

27.0

22.2

1.74

0.01

8.41

23.8

33.3

3.2

0.0

1.6

0.0

9.5

28.6

0.01

82.5

17.5

0.16

60.6

$\begin{array}{ll}79.4 & 8.68^{*} \\ 7.06^{* \star}\end{array}$

$3.2 \quad 3.61$

$14.3 \quad 0.67$

$3.2 \quad 2.74$

$30.2 \quad 0.69$

$34.9 \quad 1.10$

$67.64 .82^{*}$

$40.4 \quad 0.71$

$45.6 \quad 0.77$

$7.1 \quad 0.91$

$14.0 \quad 2.42$

Note: ABFT = attachment-based family therapy; NSSI = nonsuicidal self-injury; NST = nondirective supportive therapy. ${ }^{*} \mathrm{p}<.05 ;{ }^{* *} \mathrm{p}<.01$. 
TABLE 2 Mean and Standard Deviation for Baseline Measure $(\mathrm{N}=129)$

\begin{tabular}{|c|c|c|c|c|c|c|c|}
\hline \multirow[b]{2}{*}{ Variable } & \multicolumn{2}{|c|}{ Total $(\mathbf{N}=129)$} & \multicolumn{2}{|c|}{ ABFT $(n=66)$} & \multicolumn{2}{|c|}{ NST $(n=63)$} & \multirow[b]{2}{*}{$t$} \\
\hline & Mean & SD & Mean & SD & Mean & SD & \\
\hline SIQ Monthly & 49.89 & 15.16 & 49.49 & 14.66 & 50.27 & 15.73 & -0.29 \\
\hline BDI (adolescent) & 30.54 & 7.97 & 31.06 & 7.68 & 29.99 & 8.29 & -0.76 \\
\hline Conflict & 10.89 & 3.37 & 10.89 & 3.24 & 10.89 & 3.53 & 0.01 \\
\hline Cohesion & 14.37 & 3.37 & 14.30 & 3.34 & 14.44 & 3.43 & 0.23 \\
\hline
\end{tabular}

Note: $A B F T=$ attachment-based family therapy; BDI = Beck Depression Inventory II; NST = nondirective supportive therapy; SIQ = Suicidal Ideation Questionnaire.

weeks 24, 32, 40, and 52 but are not included in this report. Assessment staff was blinded to treatment condition.

Therapist and Therapist Training. Over a period of 4 years, 14 therapists delivered the treatments. Eleven therapists were women. Five therapists had a master's degree and 9 had doctoral degrees. To control for potential therapist effects, the same therapists delivered the 2 treatments. Therapists received training in the 2 treatments by reading the manuals, attending a 2- to 3-day training workshop, and treating pilot cases or conducting cotherapy with experienced project therapists. Then, therapists received weekly supervision from expert ABFT and NST supervisors. The 2 doctoral-level ABFT supervisors had a primary allegiance to ABFT. Of the 3 doctoral-level NST supervisors, 2 had a primary allegiance to CBT and 1 had an allegiance to couples and family therapy.

Adherence Ratings. To evaluate treatment fidelity and differentiation, raters used an adherence measure that included 22 items (17 items for tasks in ABFT and 7 items that were specific to FE-NST). Raters used a 7-point Likert scale ranging from 0 (not present) to 6 (very thorough and consistent) to rate all items for all tapes regardless of condition. After training, 5 raters blinded to treatment condition scored a total of 290 sessions, representing $20 \%$ of total sessions from 118 cases. Intraclass correlation coefficients showed a mean coder reliability with an $\alpha$ value equal to 0.88. Data showed that ABFT and NST were delivered with high fidelity (eg, treatment as intended), with $91 \%$ of tapes coded showing adherence scores higher than 4 for ABFT scales (mean 4.67, SD 6.04) and adherence scores higher than 4 for NST scales (mean 4.22, SD 4.40). For differentiation, the 7 nonspecific treatment items from NST interventions were somewhat present in ABFT as expected, but fewer than $10 \%$ of $A B F T$ items were rated higher than 0 in the NST condition.

Treatments. Informed by attachment theory, $\mathrm{ABFT}^{4}$ purports that depressive symptoms and suicidality can be precipitated, exacerbated, or buffered against by the quality of family relationships. Family problems such as high conflict, parental abdication or over-control, or more insidious traumas such as abandonment, neglect, or abuse, can rupture adolescents' confidence in parents' availability with accompanying feelings of anger and anxiety. As a result, these youth are less likely to turn to parents for help with life's challenges. In individual sessions with the adolescent, the therapist helps the teen to understand how these ruptures fuel distress, contribute to self-destructive behavior, and compromise the adolescent's ability to use parents as a resource for managing suicidal thoughts and feelings. The therapist prepares the adolescent to discuss these ruptures with the parent. The therapist also works with the parent alone to better understand how current stressors and attachment history might inhibit the parent from providing more emotionally supportive parenting. Then, in conjoint sessions, adolescents begin to express their perceived injustices and emotional disappointment, and parents are coached to acknowledge and validate the adolescents' feelings. This phase is designed to enact a corrective attachment experience. As trust and open communication improve, treatment shifts to promoting the adolescent's autonomy in domains outside the family. Although ABFT is focused on process and trauma, the manual offers structure and procedures to accomplish these treatment goals within a 16week treatment protocol.

FE-NST is a modification of the individual, supportive relationship treatment manual. ${ }^{20}$ Over 16 sessions, this therapy focuses on developing a supportive relationship between the adolescent and the therapist. Specifically, the therapist engages with the adolescent by listening, empathizing, identifying feelings, attending to affect, offering support and validation, and providing summarizing statements that might bring more meaning to the adolescent's experiences. These treatment factors, common to many psychotherapies, ${ }^{21}$ are believed to counteract the patient's feeling of thwarted belongingness, helplessness, and hopelessness by establishing a close relationship with the therapist. For this RCT, sessions with the adolescent were 
augmented with 1 conjoint parent-adolescent session to do safety planning and 4 parent education sessions without the adolescent. Parent education sessions focused on suicide risk assessment, understanding depression, advocacy and resource development, and problem solving.

\section{Primary Outcome Measures}

Suicidal Ideation. The SIQ-JR Monthly ${ }^{22}$ is a 15 -item measure assessing thoughts of suicide over the past month. Adolescents rate the frequency of suicidal thoughts on a 7 -point scale. Sum scores can range from 0 to 90 , with scores higher than 31 indicating clinical levels of ideation. Previous studies have shown that the SIQ-JR has high internal consistency $(\alpha=0.93-0.96) .{ }^{19}$ Internal consistency in this sample yielded an $\alpha$ value equal to 0.86 .

Depressive Symptoms. The BDI-II ${ }^{17}$ has 21 self-reported items assessing severity of depressive symptoms. Responses for each item are scored on a scale of 0 to 3 , with the sum of all items ranging from 0 to 63. Previous studies have shown that the BDI-II has high internal consistency $(\alpha=0.91)$ and is positively correlated with other measures of depression. In this sample, the scale demonstrated internal consistency, yielding an $\alpha$ value equal to 0.85 .

Suicide Severity. The C-SSRS ${ }^{23}$ is an interview-based measure designed to create a topology of suicidal behavior and ideation. For this study, the C-SSRS was used to identify whether the adolescent had a lifetime suicide attempt history and/or a history of nonsuicidal self-injury.

Family Conflict and Cohesion. The SRFF ${ }^{19}$ consists of 15 items measuring cohesion, conflict, and democratic family style. Previous studies have shown Cronbach $\alpha$ values ranging from 0.63 to 0.91 , with most in the 0.70 to 0.85 range. In this sample, internal consistency for the Conflict and Cohesion scales yielded $\alpha$ values of 0.70 and 0.88 , respectively.

Psychiatric Diagnoses. The DISC is a structured psychiatric diagnostic interview that evaluated more than 30 psychiatric diagnoses based on DSM-IV criteria. For the present study, the DISC modules for various anxiety disorders, mood disorders, oppositional defiant disorder, and substance abuse were administered to participating adolescents.

\section{Data Analysis}

Tests of baseline differences in demographic and clinical characteristics were conducted using independent-samples $t$ tests for continuous variables and $\chi^{2}$ or Fisher exact tests for categorical variables. Before analysis, inspection of the 2 continuous outcome measures, SIQ-JR and BDI-II, identified significant deviations from normality. Box-Cox transformations indicated that a square root transformation corrected the positive skew for the SIQ-JR and the BDI-II. Suicide attempts and nonsuicidal self-injury (measured through the C-SSRS) were treated as binary (history versus no history).

Hierarchical linear modeling tested treatment differences in rates of change across the full 16 weeks of treatment using the intent-to-treat sample. ${ }^{24}$ For the SIQ-JR and the BDI, the main efficacy analysis modeled the rate of log-linear change from baseline to 16 weeks (end of active treatment). ${ }^{25}$ All patients were included in the hierarchical linear model corresponding to a full intent-to-treat analysis. Hierarchical generalized linear modeling was used to accommodate the binary outcome (ie, suicide attempts), while accounting for clustering of repeated measures within each subject. ${ }^{26}$ Effect size was measured using the Cohen $d$ for continuous outcomes and odds ratios for the binary outcome. ${ }^{27}$ The odds ratio corresponded to, on average, the number of times an event was more likely to occur for ABFT compared with FE-NST at each assessment. Nonparametric Wilcoxon rank sum tests assessed the number of sessions attended. The $\chi^{2}$ analyses assessed treatment retention. ${ }^{28}$ The pattern-mixture model approach assessed whether missing data had a substantive influence on results. The methodology of Jacobson et al. ${ }^{29}$ was used to assess reliable change. Clinical significance was based on established thresholds for each measure. All analyses were conducted using SAS 9.4 (SAS Institute, Cary, NC).

\section{Statistical Power}

Using the method described by Diggle et al. ${ }^{30}$ and Ahn et al., ${ }^{31}$ power formulas for linear mixed models with repeated measures showed that the analysis of the SIQ-JR was well powered with a targeted sample size of $110 \mathrm{pa}-$ tients ( 55 completers per group). A priori, using an $\alpha$ level at 0.05 based on a 2-tailed test and assuming a within correlation of 0.5 with 4 post-baseline assessments, we had power of $91.2 \%$ and $80.0 \%$ to detect a betweenintervention effect size of 0.50 and 0.42 , respectively. In addition, the study had greater than $80 \%$ power to detect a difference in the proportion of suicide attempts (yes versus no) of at least one-sixth.

\section{RESULTS}

\section{Treatment Retention}

There was no significant difference between the number of sessions attended by ABFT participants (mean 14.34, SD 7.58) and FE-NST participants (mean 12.67, SD 5.74; $t_{127}=-1.43, p=.16$ ). There was no significant difference between the number of weeks attended by patients in the 2 treatments (ABFT, mean 9.68, SD 4.72; FE-NST, mean 9.43, SD 4.20; $\left.t_{127}=-0.32, p=.75\right)$. Dropout rates were 
not significantly different between treatment groups, with $18.2 \%$ attrition for ABFT compared with $17.5 \%$ for FENST $\left(\chi^{2}[1, N=129]=0.02, p=.92\right.$; Figure 1$)$.

\section{Suicide Ideation}

There was no significant between-group difference in the rate of change in self-reported ideation (SIQ-JR; $F_{1,127}=$ $1.81, p=.18$; Figure 2). The estimated total change from baseline to end of treatment (week 16) was -31.55 (SE 2.50) for ABFT and -27.42 (SE 2.52) for FE-NST. On average, ABFT participants showed a significant decrease in suicidal ideation $\left(t_{127}=12.61, p<.0001\right.$; effect size, $d=$ 2.24). Similarly, participants in the FE-NST showed a significant decrease in suicidal ideation $\left(t_{127}=10.88, p<\right.$ .0001 ; effect size, $d=1.93$ ).

Remission (below clinical cutoff) rates in suicide ideation (ie, SIQ-JR score < 12) were $14.3 \%$ for ABFT versus $12.1 \%$ for FE-NST at 4 weeks, $25.0 \%$ for ABFT versus $11.5 \%$ for FE-NST at 8 weeks, $24.5 \%$ for ABFT versus $26.5 \%$ for FENST at 12 weeks, and $32.7 \%$ for ABFT versus $24.5 \%$ for FE-NST at post-treatment (16 weeks). There were no significant between-treatment differences in remission rates over the 16 weeks of treatment.

Response rates (ie, $\geq 50 \%$ decrease in SIQ-JR score from baseline) were $32.1 \%$ for ABFT versus $32.8 \%$ for FE-NST at 4 weeks, $51.9 \%$ for ABFT versus $36.5 \%$ for FE-NST at 8 weeks, $58.5 \%$ for ABFT versus $51.0 \%$ for FE-NST at 12 weeks, and $69.1 \%$ for ABFT versus $62.3 \%$ for FE-NST at post-treatment (16 weeks). The differences in treatment response rates between the 2 conditions were not significant. Reliable change in SIQ-JR score required difference scores of at least 23.02. The reliable change was $56.6 \%$ for ABFT versus $49.2 \%$ for FE-NST at post-treatment (week 16), with no significant differences between the 2 conditions.

\section{Depressive Symptoms}

There was no difference between the 2 treatment conditions in the rate of change in depression (BDI-II) over the course of treatment $\left(F_{1,127}=0.11, p=.74\right.$; Figure 3$)$. The estimated total change from baseline to end of treatment was -5.40 (SE 0.50) for ABFT and -4.87 (SE 0.50) for FE-NST on the BDI-II. Participants in the ABFT group showed a significant decrease in BDI-II score $\left(t_{127}=10.72\right.$, $p<.0001$; effect size, $d=1.90)$. Similarly, FE-NST participants showed a significant decrease in BDI-II score $\left(t_{127}=9.68, p<.0001\right.$; effect size, $\left.d=1.72\right)$.

Remission rates in depressive symptoms (ie, BDI-II score $<9)^{32}$ were $7.1 \%$ for ABFT versus $15.5 \%$ for FENST at week $4,19.2 \%$ for ABFT versus $11.8 \%$ for FENST at week $8,35.9 \%$ for ABFT versus $18.4 \%$ for FE-NST at week 12 , and $40.0 \%$ for ABFT versus $34.0 \%$
FIGURE 2 Suicide Ideation by Treatment Condition at Weeks $0,4,8,12$, and 16

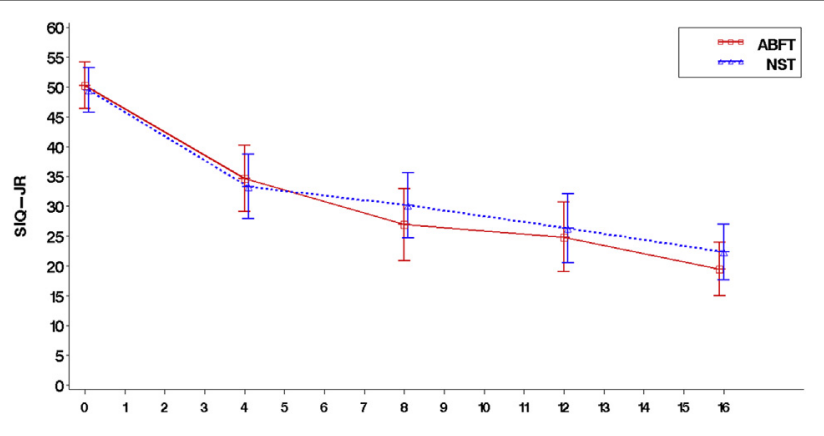

Note: $A B F T=$ attachment-based family therapy; NST = nondirective supportive therapy; $S I D-J R=$ Suicidal Ideation Questionnaire-Jr. Please note color figures are available online.

for FE-NST at post-treatment (week 16). A significant between-treatment difference in response rates per depressive symptoms was seen at 12 weeks favoring ABFT over FE-NST (odds ratio 2.88, 95\% CI 1.10-7.60; $\chi_{1}^{2}=4.60$, $p=.03)$. Reliable change in BDI-II score required difference scores of at least 12.10 . These rates were $70.9 \%$ for ABFT versus $56.6 \%$ for FE-NST at post-treatment (week 16). There were no significantly different reliable change scores between treatment conditions across the treatment course.

\section{Suicide Attempts}

During the course of treatment, 6 of the 129 adolescents reported making a suicide attempt during the treatment phase. Of those 6, 2 were enrolled in ABFT (3.0\%) and 4 were enrolled in FE-NST (6.4\%), with no significant difference between groups $\left(\chi_{1}^{2}=0.80, p=.37\right)$. Similar to other suicide attempter studies, we also looked at patients who came to the study after a recent suicide attempt and hospitalization $(\mathrm{n}=51)$. Of these recent attempters, 2 were

\section{FIGURE 3 Depressive Symptoms by Treatment at Weeks 0,} $4,8,12$, and 16

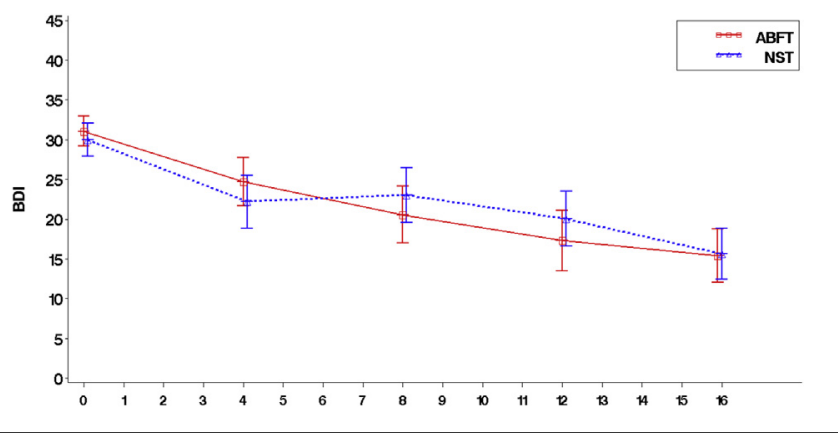

Note: $A B F T=$ attachment-based family therapy; BDI = Beck Depression Inventory II; NST = nondirective supportive therapy. Please note color figure are available online.

Journal of the American Academy of Child \& Adolescent Psychiatry Volume 58 / Number 7 / July 2019 
from the ABFT group (6.9\%) and 2 were from the FE-NST group $(9.1 \%)$, with no significant difference between groups $\left(\chi_{1}^{2}=0.08, p=.77\right)$.

\section{DISCUSSION}

This is the first RCT comparing ABFT with an active, rigorously controlled comparison therapy for treating suicide ideation and depressive symptoms in adolescents. Although adolescents in ABFT and FE-NST showed substantial decreases in suicide ideation and depressive symptoms, there were no differences in symptom decrease between the 2 treatment conditions. On average, suicide ideation decreased 24 points across the 2 treatments, allowing many adolescents to move into a normative range below 12 on the SIQ (35\% for ABFT, 28\% for FE-NST), with an overall clinical response rate of $69 \%$ for ABFT and $62 \%$ for FE-NST patients. Rates of suicide attempts after admission to the trial were low even among adolescents who had made a recent suicide attempt. These findings might reflect the efficacy of these 2 relatively brief, low-dose outpatient treatments for adolescents referred for serious suicidal thoughts and behaviors. Alternatively, because depression and suicide ideation are time-sensitive symptoms, these outcomes could be influenced by regression to the mean over the 16 weeks of treatment.

Comparisons with other treatment studies are complicated by heterogeneity in sample demographics, inclusion criteria, and outcome measures and by treatment elements and duration. Nevertheless, some comparison on suicide attempts and attrition is informative. Of those patients starting treatment soon after a suicide attempt $(\mathrm{n}=51), 6.9 \%$ of ABFT participants and $9.1 \%$ of FENST participants made an attempt during the treatment phase. This compares favorably to a meta-analysis that failed to find significant decreases in suicide attempts when active interventions were compared with TAU. ${ }^{33,34}$ In the Treatment Study of Adolescent Suicide Attempters (TASA) ${ }^{35}$ consisting of medication plus CBT plus family, $12 \%$ of participants made an attempt within 6 months. ${ }^{36}$ In another study, $25 \%$ of participants in a skills training condition and $12 \%$ in an NST condition reattempted suicide during the treatment phase. For retention, attrition from ABFT was $18.2 \%$ and attrition from FE-NST was $17.5 \%$, comparable to similar studies. ${ }^{11,35}$ For treatment dose, ABFT was delivered as a single modality by a community therapist, generally once a week (mean 14 sessions). This contrasts with many other studies that use a multicomponent treatment protocol (eg, individual and group therapy plus medication), multiple contact hours a week, and delivery by whole teams of expert therapists. Thus, the structure of ABFT and NST might fit well within typical outpatient treatment settings. Future comparisons across studies would be strengthened if they shared common measurement tools and agreed on definitions of remission, response, clinical significance, adequate dose, and attrition.

In addition to treatment dose and length of treatment, which were controlled in the 2 conditions, other common factors could account for the equivalent efficacy of ABFT and FE-NST. First, all families entered treatment at moments of high crisis and were met with supportive staff and therapists who offered immediate access to treatment, a 24hour available crisis hotline, weekly check-in phone calls, home visits as needed, and assistance with social services, school, or medication consults. These treatment features and special attention to retention should be considered essential and best practice for this population. The responsiveness of the program alone could have accounted for some of the treatment response and low rates of suicide attempts in the study. ${ }^{37}$ Second, the therapist crossover design and high treatment adherence provided consistent empathy, support, and treatment fidelity and controlled for the possible confound of treatment allegiance, all of which have been associated with treatment outcome. ${ }^{11}$ Third, the 2 treatments shared many common elements, including family safety planning, parent involvement, and parent psychoeducation and support, validation, and empathy in individual sessions with adolescents and parent(s) alone. ${ }^{20}$ Fourth, although investigators usually view NST as a control treatment, it has proved equivalent to CBT in a metaanalysis of treatment studies for adults with depression after controlling for treatment allegiance. ${ }^{12}$

Treatment outcomes also might have resulted from mechanisms specific to ABFT and FE-NST. ${ }^{38}$ In ABFT, the primary aim is to improve trust and communication in the parent-adolescent dyad. These improvements are hypothesized to lessen family conflict and emotional chaos and increase an adolescent's reliance on parents as a safety net. Addressing these attachment ruptures can lead to more coherent (ie, secure) attachment schemas and a variety of mental health benefits (eg, improved self-worth, better emotion regulation). Possibly ABFT would be fortified by integrating more specific cognitive and emotional skills training (eg, parent or adolescent psychoeducation, CBT, or dialectical behavioral therapy techniques). Thus, building a more multicomponent $\mathrm{ABFT}$ treatment program might be worthy of investigation.

Alternatively, NST focuses on the therapist establishing a supportive relationship with the adolescent through reflective and empathic listening. This clinical process is similar to mentalization-based therapy, which focuses on reflective conversations that are alleged to change the 
adolescent's self-understanding, sense of agency, and selfregulatory capacities. ${ }^{39}$ In NST the adolescent's relationship with the therapist is primary and was evident in higher ratings of the therapeutic alliance in the NST than in the ABFT condition. ${ }^{40}$ These findings suggest that ABFT might be potentiated by increasing the amount of individual time devoted to the adolescent and adding individual skill training or medication elements to the treatment protocol. Building a more multicomponent treatment program might be worthy of investigation.

The failure to confirm the superiority of ABFT over FE-NST is consistent with many RCTs of 2 active treatments that fail to demonstrate the superiority of a specific treatment. ${ }^{41,42}$ However, there are several notable limitations to the present findings. First, although nearly $70 \%$ of patients reported clinically significant change, only $40 \%$ obtained remission (nonclinical symptoms) by the end of 16 weeks. Some patients might have needed a longer treatment, additional treatments, or another type of treatment. Second, the 2 treatments were delivered in a highly regarded children's hospital and university, which engenders the kind of institutional alliance that has often been associated with better outcomes. ${ }^{43}$ Testing these treatments in real-world clinical settings might be needed to evaluate their true clinical value. ABFT has shown some promise in this area. ${ }^{9,10}$ Future examination of long-term outcomes, treatment moderators and mediators, and relapse might help

\section{REFERENCES}

1. Anderson RN. Deaths: leading causes for 2000. Natl Vital Stat Rep. 2002;50:1-85.

2. Glenn CR, Franklin JC, Nock MK. Evidence-based psychosocial treatments for selfinjurious thoughts and behaviors in youth. J Clin Child Adolesc Psychol. 2015; 44:1-29.

3. Spirito A, Stanton C, Donaldson D, Boergers J. Treatment-as-usual for adolescent suicide attempters: implications for the choice of comparison groups in psychotherapy research. J Clin Child Adolesc Psychol. 2002;31:41-47.

4. Diamond GS, Diamond GM, Levy SA. Attachment-Based Family Therapy for Depressed Adolescents. Washington, DC: American Psychological Association; 2014.

5. Brent DA, Holder D, Kolko D, et al. A clinical psychotherapy trial for adolescent depression comparing cognitive, family, and supportive therapy. Arch Gen Psychiatry. 1997;54:877-885.

6. Zisk A, Abbott CH, Ewing SK, Diamond GS, Kobak R. The Suicide Narrative Interview: adolescents' attachment expectancies and symptom severity in a clinical sample. Attach Hum Dev. 2017;19:447-462.

7. Kobak R, Zajac K, Herres J, Krauthamer Ewing ES. Attachment based treatments for adolescents: the secure cycle as a framework for assessment, treatment and evaluation. Attach Hum Dev. 2015;17:220-239.

8. Diamond GS, Wintersteen MB, Brown GK, et al. Attachment-based family therapy for adolescents with suicidal ideation: a randomized controlled trial. J Am Acad Child Adolesc Psychiatry. 2010;49:122-131.

9. Israel P, Diamond GS. Feasibility of attachment based family therapy for depressed clinic-referred Norwegian adolescents. Clin Child Psychol Psychiatry. 2013;18:334-350.

10. Santens T, Devacht I, Dewulf S, Hermans G, Bosmans G. Attachment-based family therapy between Magritte and Poirot: dissemination dreams, challenges, and solutions in Belgium. Aust N Z J Fam Ther. 2016;37:240-250.

11. Donaldson D, Spirito A, Esposito-Smythers C. Treatment for adolescents following a suicide attempt: results of a pilot trial. J Am Acad Child Adolesc Psychiatry. 2005;44: 113-120. determine what kind of patients responded best to which treatment.

\section{Accepted October 22, 2018.}

Drs. Diamond, Krauthamer Ewing, and Levy are with the Center for Family Intervention Science, Drexel University, Philadelphia, PA. Dr. Kobak is with the University of Delaware, Newark. Dr. Herres is with The College of New Jersey, Ewing Township. Dr. Russon is with Virginia Tech, Blacksburg. Dr. Gallop is with the Applied Statistics Program, West Chester University, PA.

This research was supported by National Institute of Mental Health grant 5R01MH091059-03 (Dr. Diamond and Dr. Kobak, co-principal investigators). The trial was prospectively registered at the US National Institutes of Health clinicaltrials.gov registry (NCT01537419)

Dr. Gallop served as the statistical expert for this research.

The authors thank the dedicated staff who worked tirelessly on this project, especially Linda Boamah-Wiafe, BA, of the University of Connecticut; Margot Adams, MSW, of Stanford University; Tamar Kodish, BA, of the University of California-Los Angeles; and Annie Shearer, BA, of the University of Pittsburgh School of Medicine. They also sincerely thank all the study participants and collaborating referral providers, including the network of primary care clinicians, patients, and families from the Pediatric Research Consortium (PeRC) at The Children's Hospital of Philadelphia.

Disclosure: Drs. Diamond and Levy have received funding from the National Institute of Mental Health. They have received royalties from AttachmentBased Family Therapy (2014) book sales and honoraria for ABFT trainings and supervision. Drs. Kobak, Ewing, Herres, Russon, and Gallop have received funding from the National Institute of Mental Health.

Correspondence to Guy S. Diamond, PhD, Center for Family Intervention Science, College of Nursing and Health Professions, Drexel University, 3020 Market Street, Philadelphia, PA, 19072; e-mail: guy.diamond@drexel.edu

0890-8567/\$36.00/@2018 American Academy of Child and Adolescent Psychiatry

https://doi.org/10.1016/j.jaac.2018.10.006
12. Cuijpers P, Driessen E, Hollon SD, van Oppen P, Barth J, Andersson G. The efficacy of non-directive supportive therapy for adult depression: a meta-analysis. Clin Psychol Rev. 2012;32:280-291.

13. Prinstein MJ, Nock MK, Simon V, Aikins JW, Cheah CSL, Spirito A. Longitudinal trajectories and predictors of adolescent suicidal ideation and attempts following inpatient hospitalization. J Consult Clin Psychol. 2008;76:92-103.

14. Spirito A, Esposito-Smythers C. Attempted and completed suicide in adolescence. Annu Rev Clin Psychol. 2006;2:237-266.

15. Joe S, Baser RE, Breeden G, Neighbors HW, Jackson JS. Prevalence of and risk factors for lifetime suicide attempts among blacks in the United States. JAMA. 2006;296: 2112-2123.

16. Miranda J, Nakamura R, Bernal G. Including ethnic minorities in mental health intervention research: a practical approach to a long-standing problem. Cult Med Psychiatry. 2003;27:467-486.

17. Beck AT, Steer RA, Ball R, Ranieri W. Comparison of Beck Depression Inventories-IA and -II in psychiatric outpatients. J Pers Assess. 1996;67:588-597.

18. Shaffer D, Fisher P, Lucas CP, Dulcan MK, Schwab-Stone ME. NIMH Diagnostic Interview Schedule for Children Version IV (NIMH DISC-IV): description, differences from previous versions, and reliability of some common diagnoses. J Am Acad Child Adolesc Psychiatry. 2000;39:28-38.

19. Reynolds WM, Mazza JJ. Reliability and validity of the Reynolds Adolescent Depression Scale with Young Adolescents. J Sch Psychol. 1998;36:295-312.

20. Brent DA, Kolko DJ. Supportive Relationship Treatment Manual. Pittsburgh, PA: University of Pittsburgh/WPIC, Department of Psychiatry; 1991.

21. Sprenkle DH, Davis SD, Lebow J. Common Factors in Couple and Family Therapy. New York: Guilford Press; 2009.

22. Reynolds WM, Mazza JJ. Assessment of suicidal ideation in inner-city children and young adolescents: reliability and validity of the Suicidal Ideation Questionnaire-JR. Sch Psychol Rev. 1999;28:17-30. 
23. Posner K, Brown GK, Stanley B, et al. The Columbia-Suicide Severity Rating Scale: initial validity and internal consistency findings from three multisite studies with adolescents and adults. Am J Psychiatry. 2011;168:1266-1277.

24. Gallop R, Tasca GA. Multilevel modeling of longitudinal data for psychotherapy researchers: II. The complexities. Psychother Res. 2009;19:438-452.

25. Gallop RJ, Dimidjian S, Atkins DC, Muggeo V. Quantifying treatment effects when flexibly modeling individual change in a nonlinear mixed effects model. J Data Sci. 2011;9:221-241

26. Tate R. Interpreting hierarchical linear and hierarchical generalized linear models with slopes as outcomes. J Exp Educ. 2004;73:71-95.

27. McGough JJ, Faraone SV. Estimating the size of treatment effects: moving beyond $\mathrm{p}$ values. Psychiatry (Edgmont). 2009;6:21-29.

28. Onchiri S. Conceptual model on application of chi-square test in education and social sciences. Educ Res Rev. 2013;8:1231-1241.

29. Jacobson NS, Roberts LJ, Berns SB, McGlinchey JB. Methods for defining and determining the clinical significance of treatment effects: description, application, and alternatives. J Consult Clin Psychol. 1999;67:300-307.

30. Diggle P, Liang K-Y, Zeger SL. Analysis of Longitudinal Data. Oxford, UK: Clarendon Press; New York: Oxford University Press; 1994.

31. Ahn C, Overall JE, Tonidandel S. Sample size and power calculations in repeated measurement analysis. Comput Methods Programs Biomed. 2001;64:121-124.

32. Riedel M, Möller H-J, Obermeier M, et al. Response and remission criteria in major depression-a validation of current practice. J Psychiatr Res. 2010;44: 1063-1068.

33. Ougrin D, Tranah T, Stahl D, Moran P, Asarnow JR. Therapeutic interventions for suicide attempts and self-harm in adolescents: systematic review and meta-analysis. J Am Acad Child Adolesc Psychiatry. 2015;54:97-107.e2.
34. Asarnow JR, Hughes JL, Babeva KN, Sugar CA. Cognitive-behavioral family treatment for suicide attempt prevention: a randomized controlled trial. J Am Acad Child Adolesc Psychiatry. 2017;56:506-514.

35. Vitiello B, Brent DA, Greenhill LL, et al. Depressive symptoms and clinical status during the Treatment of Adolescent Suicide Attempters (TASA) study. J Am Acad Child Adolesc Psychiatry. 2009;48:997-1004.

36. Brent DA. The Treatment of SSRI-Resistant Depression in Adolescents (TORDIA): in search of the best next step. Depress Anxiety. 2009;26:871-874

37. Brent DA, McMakin DL, Kennard BD, Goldstein TR, Mayes TL, Douaihy AB. Protecting adolescents from self-harm: a critical review of intervention studies. J Am Acad Child Adolesc Psychiatry. 2013;52:1260-1271

38. DeRubeis RJ. A conceptual and methodological analysis of the nonspecifics argument Clin Psychol. 2005;12:174-183.

39. Allen JG, Association AP. Restoring Mentalizing in Attachment Relationships: Treating Trauma with Plain Old Therapy. Arlington, VA: American Psychiatric Publishers; 2013

40. Ibrahim M, Jin B, Russon J, Diamond G, Kobak R. Predicting alliance for depressed and suicidal adolescents: the role of perceived attachment to mothers. Evid Based Pract Child Adolesc Ment Health. 2018;3:42-56.

41. Goodyer IM, Reynolds S, Barrett B, et al. Cognitive behavioural therapy and short-term psychoanalytical psychotherapy versus a brief psychosocial intervention in adolescents with unipolar major depressive disorder (IMPACT): a multicentre, pragmatic, observerblind, randomised controlled superiority trial. Lancet Psychiatry. 2017;4:109-119.

42. Wampold BE, Minami T, Baskin TW, Callen Tierney S. A meta-(re)analysis of the effects of cognitive therapy versus "other therapies" for depression. J Affect Disord. 2002;68:159-165.

43. Flückiger C, Del Re AC, Wampold BE, Symonds D, Horvath AO. How central is the alliance in psychotherapy? A multilevel longitudinal meta-analysis. J Couns Psychol. 2012;59:10-17. 